\title{
Risk of Potential Glucocorticoid-Related Adverse Events in Patients with Giant Cell Arteritis: Results from a USA-Based Electronic Health Records Database
}

\author{
Jennie H. Best · Amanda M. Kong (D) - Sebastian Unizony • \\ Oth Tran · Margaret Michalska
}

Received: September 26, 2019 / Published online: October 26, 2019

(C) The Author(s) 2019

\begin{abstract}
Introduction: Oral glucocorticoids (GC) have been the mainstay of treatment for giant cell arteritis (GCA). We estimated the risk and dose-effect relationship of potential GC-related adverse events (AEs) in patients with GCA.

Methods: This retrospective, observational cohort study utilized data from the IBM Explorys Electronic Health Records database from 2008 through 2016. Inclusion criteria included the presence of at least two GCA diagnostic codes in subjects aged 50 or older along with supporting laboratory [C-reactive protein (CRP) or erythrocyte sedimentation rate (ESR)], prescription data on oral GCs, and at least
\end{abstract}

Enhanced Digital Features To view enhanced digital features for this article go to https://doi.org/10.6084/ m9.figshare.9980936.

J. H. Best · M. Michalska

Genentech, Inc., 1 DNA Way, South San Francisco, CA, USA

A. M. Kong $(\bowtie) \cdot$ O. Tran

IBM Watson Health, 75 Binney Street, Cambridge, MA, USA

e-mail: akong@us.ibm.com

S. Unizony

Massachusetts General Hospital, Harvard Medical

School, 55 Fruit Street, Boston, MA, USA
12 months of follow-up before and after the first oral GC prescription for GCA (index date). Potential AEs captured on the basis of new diagnoses, prescriptions, and laboratory tests were assessed during the 12 months post-index date. Results were descriptively summarized across cohorts according to quartiles (Q) of mean daily GC dose measured over the first 6 months of follow-up (Q1, $\geq 1.00$ to $\leq 13.75 \mathrm{mg} ; \mathrm{Q} 2,>13.75$ to $\leq 25.00 \mathrm{mg} ; \mathrm{Q} 3$, $>25.00$ to $\leq 40.00 \mathrm{mg}$; Q4, > $40.00 \mathrm{mg}$ ).

Results: We identified 785 eligible patients with GCA. The mean (SD) age of the cohort was 76 (9) years and $70 \%$ were female. The mean oral GC dose during the first 6 months post-index was $28.9 \mathrm{mg} /$ day. A dose-effect response was observed from Q1 to Q4 in the following potential GC-related AEs: newly diagnosed type 2 diabetes/HbA1c $>7.5 \%$ (range $7.5-24.5 \%$ ), blood glucose $\geq 200 \mathrm{mg} / \mathrm{dL}$ (range $7.5-15 \%$ ), serious infection (range 16.8-24.8\%), cataracts (range 12.0-21.7\%), gastrointestinal bleed/ulcer (range 6.0-11.8\%), and increase in $\mathrm{BMI} \geq 5$ units (range 4.1-6.4).

Conclusions: In patients with GCA, potential GC-related AEs increased with higher daily oral GC doses. This highlights the need for effective therapies that reduce GC exposure and toxicity. Funding: Genentech, Inc.

Keywords: Adverse events; Electronic health records; Giant cell arteritis; Glucocorticoids 


\section{Key Summary Points}

\section{Why carry out this study?}

Patients with giant cell arteritis (GCA), a systemic inflammatory disease commonly presenting with persistent, severe headache, are typically treated with high doses of oral glucocorticoids; however, oral glucocorticoids are associated with several adverse events.

We sought to evaluate the prevalence of adverse events (AEs) among patients with GCA treated with various doses of oral glucocorticoids using a US electronic health records database with interest in investigating the relationship between AEs and glucocorticoid dosing.

\section{What was learned from this study?}

The proportion of patients experiencing AEs, such as incident type 2 diabetes, hyperglycemia, serious infection, cataract, increase in BMI, and gastrointestinal bleed or ulcer, increased as the average daily dose of oral glucocorticoids increased.

This highlights the need for effective therapies to reduce glucocorticoid exposure and toxicity in patients with GCA.

\section{INTRODUCTION}

Giant cell arteritis (GCA) is a vasculitis of medium- and large-sized arteries. Typical clinical manifestations of GCA include new onset headache, scalp tenderness, jaw pain, visual symptoms (including blindness), shoulder and hip pain and stiffness, and constitutional complaints (e.g., fever and weight loss) [1]. GCA is the most common type of primary vasculitis with a projected prevalence of approximately 950,000 cases in the USA by 2050 [2]. The disease is more common in women over 50 years of age [3], with an age- and sex-adjusted prevalence rate of 204 per 100,000 population [4].

Until recently, lengthy tapers of oral glucocorticoids (GC) have been the mainstay of treatment for GCA [5]. Despite their established therapeutic role, prolonged GC exposure leads to toxicity including serious adverse events (AE) in the majority of patients with GCA [6-14]. GC-related side effects are diverse and may include diabetes, hypertension, osteoporosis, fragility fractures, cataracts, glaucoma, gastrointestinal bleeding, psychosis, weight gain, insomnia, and infection [6-14].

Several studies have investigated the risk of AEs associated with GC use among patients with GCA [7-14]. In a retrospective analysis of 2497 patients with GCA and 9680 patient-years of follow-up, GC treatment was associated with a significant increase in the development of metabolic bone disease, cataracts, glaucoma, pneumonia, and new onset diabetes [7]. A recent claims-based study from the USA and the UK confirmed these findings, noting that the risk of GC-related AEs increased with progressively higher cumulative GC exposures [12].

The relationship between daily GC dosing and GC-related toxicity has been incompletely studied. In addition, to date, most studies of GC-related AEs have been limited by the use of administrative claims databases. These databases typically do not include prescription data and laboratory results, which can be useful in identifying AEs and characterizing their severity (e.g., hemoglobin A1c [HbA1c] for evaluation of AEs related to glucose metabolism).

The aims of this study were to assess the realworld use of GC in GCA, quantify the risk of GC-related toxicity in this patient population, and establish the dose-effect relationship between daily GC dosing and GC-related AEs comprehensively defined on the basis of diagnostic codes, laboratory, and prescription data.

\section{METHODS}

\section{Study Design}

This retrospective, observational cohort study utilized US electronic health records (EHR) data 
from the IBM Explorys ${ }^{\circledR}$ Database between January 1, 2008 and December 31, 2016. We identified incident cases of GCA and characterized their GC treatment. Eligible patients were stratified into four quartiles based on their mean daily oral GC dose. The index date was set as the date of the first prescription for oral GC occurring within 6 months following the GCA diagnosis. The study period consisted of a 12-month pre-index baseline period over which baseline clinical characteristics were measured, and a 12-month post-index follow-up period during which the study outcomes were assessed.

\section{Data Source}

IBM Explorys is a computerized medical database that contains longitudinal EHR data of approximately 55 million patients from 23 integrated health networks composed of nearly 360 hospitals and 330,000 providers. The database contains information regarding health care provided in the inpatient, ambulatory, emergency, and post-acute settings. The data include diagnoses (International Classification of Diseases-Clinical Modification [ICD-CM] Version 9 or 10, Systematized Nomenclature of Medicine [SNOMED], procedures (Current Procedure Terminology [CPT], Healthcare Common Procedure System [HCPCS], Berenson-Eggers Type of Service [BETOS], SNOMED), medications (National Drug Code [NDC], RxNorm, SNOMED), laboratory test results (Logical Information Identifiers Names and Codes [LOINC]), patientreported outcomes, vital signs, body mass index (BMI), encounter-level information, providers (National Provider Indication [NPI], National Plan and Provider Enumeration System [NPPES]), and other clinical, financial, and operational data.

All database records are anonymized and fully compliant with US patient confidentiality requirements, including the Health Insurance Portability and Accountability Act (HIPAA) of 1996. Because this study did not involve the collection, use, or transmittal of individually identifiable data, institutional review board (IRB) approval was not required.

\section{Study Population}

The study population comprised adult patients aged 50 years or older with at least two diagnoses (inpatient or outpatient) for GCA (ICD-9CM code 446.5 or ICD-10-CM code M31.5 or M31.6) within 7-90 days of one another between January 1, 2009 and December 31, 2015. The definition of GCA also required at least two prescriptions for oral GC within 6 months of the first GCA diagnostic code, and one or more C-reactive protein (CRP) or erythrocyte sedimentation rate (ESR) laboratory test during the 12 months preceding the GCA diagnosis. The index date was defined as the date of the first GC prescription occurring on or after the qualifying GCA diagnosis. Additionally, patients were required to have at least one encounter in the 90 days before index date to indicate data availability in the pre-index period (i.e., baseline period) as well as at least one encounter in the 90 day- 12 months after the index date to indicate data availability in the post-index period. Patients with evidence of an autoimmune disease (i.e., ankylosing spondylitis, Crohn's disease, polyarteritis nodosa, psoriatic arthritis, rheumatoid arthritis, ulcerative colitis, granulomatosis with polyangiitis, systemic lupus erythematosus) or a disease requiring high-dose GC (i.e., asthma, multiple sclerosis) any time during the 12-month pre-index period or up to 24 months post-index were excluded. Patients who had anti-TNF exposure (adalimumab, certolizumab, etanercept, golimumab, or infliximab) in the 12-month pre-index period or exposure to tocilizumab during the 12-month pre-index through 24 months after the index date were also excluded.

\section{Assessment of Oral Glucocorticoid Exposure}

Patients were classified into one of four quartiles (Q) according to their mean oral GC daily dose over 6 months post-index. The daily dose of oral GC was calculated using one of the following two formulae depending on the availability of required information within the data: daily 
dose $=($ strength $\times$ number of tablets $\times$ frequency per day) $\times$ prednisone conversion factor; or daily dose $=[$ (quantity of pills $\times$ dose strength $\times$ number of refills)/(end date - start date +1 day) $] \times$ prednisone conversion factor. The dosing distribution quartiles were as follows: $\mathrm{Q} 1, \geq 1.00$ to $\leq 13.75 \mathrm{mg} /$ day; $\mathrm{Q} 2,>13.75$ to $\leq 25.00 \mathrm{mg} /$ day; $\mathrm{Q} 3,>25.00$ to $\leq 40.00 \mathrm{mg} /$ day; and Q4, > $40.00 \mathrm{mg} /$ day. Among patients whose dose was at least $40 \mathrm{mg} /$ day on or within 30 days after the index date, those with an eventual decrease in dose to below $40 \mathrm{mg} /$ day were identified. Additionally, patients with evidence of an oral GC daily dose increase after a previous decrease were also captured.

\section{Outcomes}

Potential GC-related AEs assessed during the 12-month post-index period included bone-related events (i.e., osteoporosis, fracture [vertebral and non-vertebral], and new prescriptions for osteopenia/osteoporosis [e.g., bisphosphonates]), endocrine/metabolic events (i.e., new onset type 2 diabetes [T2D] based on diagnosis and hemoglobin A1c [HbA1c] $\geq 7.5 \%$, worsening $\mathrm{T} 2 \mathrm{D}$, defined as HbA1c $\geq 7.5 \%$ in the post-index period but not in the baseline period for patients with pre-existing T2D, blood glucose level $\geq 200 \mathrm{mg} / \mathrm{dL}$, oral glucose tolerance test [OGTT] $\geq 200 \mathrm{mg} / \mathrm{dL}$, diagnosis of impaired fasting glucose or glucose intolerance, obesity diagnosis, increase in BMI $\geq 5$ units based on BMI calculated from patient records and recorded under BMI-specific LOINC codes, adrenal insufficiency), serious infections (defined as hospitalization with a diagnosis of infection or receipt of intravenous antibiotics), hospitalization with myocardial infarction diagnosis, hospitalization with stroke diagnosis, gastrointestinal (GI) events (i.e., GI bleeding or ulcer), and ophthalmologic events (i.e., cataracts or glaucoma). The occurrence of AEs was summarized across the four daily oral GC dose quartiles.

\section{Covariate Assessment}

Demographic characteristics were measured on the index date and included age, sex, and geographical region. Baseline comorbid conditions were identified on the basis of diagnoses present on medical claims during the 12-month pre-index period. These included the Deyo-Charlson Comorbidity Index (DCCI) and diagnoses based on the DCCI (i.e., mild to moderate diabetes, cerebrovascular disease, rheumatologic disease other than systemic lupus erythematosus and rheumatoid arthritis [specifically polymyositis, Felty's syndrome, rheumatoid lung, and polymyalgia rheumatica], renal disease, chronic pulmonary disease, congestive heart failure, malignancy, peripheral vascular disease, and diabetes with chronic complications). The severity of GCA was determined by the presence of a diagnosis of vision impairment (including blindness) and diagnosis of aortic aneurysm during the baseline period. CRP and ESR values were based on laboratory tests conducted during the 12 months pre- and post-index periods.

\section{Statistical Analyses}

All data including demographics, comorbidities, oral GC prescription patterns, and AEs (stratified by the four GC quartiles) were analyzed using descriptive methods. Mean and standard deviation (SD) were reported for continuous variables and counts and percentages were reported for categorical variables. We report trends in outcomes across quartiles; no statistical comparisons were made between the dosing quartiles. Additionally, patients for whom the GC dose could not be calculated were excluded from the AE analysis $(n=58)$.

\section{RESULTS}

\section{Baseline Patient Characteristics}

Of the 3968 patients diagnosed with GCA during January 1, 2009 to December 31, 2015, 785 $(19.8 \%)$ met the study definition of GCA and had 12 months of data available before and after the index date (Fig. 1). The mean age of the patients was 75.8 years $(\mathrm{SD}=8.6)$ and $69.6 \%$ were female. Table 1 summarizes other baseline 
Patients with $\geq 2$ diagnoses (inpatient or outpatient) for GCA between 1/1/2009 to $12 / 31 / 2015$ separated by $\geq 7$ days but $<90$ days; with $\geq 1$ oral GC drug prescription within 6 months of diagnosis date (index date) and age 50 years or older as of index date

$$
N=2259(100 \%)
$$

$\begin{aligned} & \text { 21 encounter in database within } 90 \text { days prior to index date, no anti-TNF exposure during } \\ & \text { the 12-month pre-index period, and second OGC prescription during the first } 6 \text { months } \\ & \text { after the diagnosis date }\end{aligned}$
No diagnoses of other autoimmune conditions or diseases associated with high
corticosteroid use and no tocilizumab use anytime during the 12-month pre-index or
24 months after index date
$\mathrm{N}=1112$ (72.4\%)
ESR laboratory test during the 12 -month pre-diagno sis period
$\mathrm{N}=785$ ( $70.6 \%)$

Fig. 1 Patient selection. CRP c-reactive protein, ESR erythrocyte sedimentation rate, GC glucocorticoid, GCA giant cell arteritis, ICD-9-CM International Classification

characteristics of this patient cohort. The mean DCCI at baseline was $1.6(\mathrm{SD}=2.0)$. The most common baseline comorbid conditions based on those included in the DCCI were mild to moderate diabetes $(24.7 \%)$, cerebrovascular disease $(19.1 \%)$, rheumatologic disease $(18.9 \%)$ primarily in the form of polymyalgia rheumatica (17.2\%), chronic pulmonary disease $(11.1 \%)$, and renal disease (11.3\%). Vision impairment including blindness was diagnosed in $11.3 \%$ of the patients; aortic aneurysms were infrequently diagnosed $(2.4 \%)$ in the pre-index period. During the 12-month pre-index period, $72.7 \%$ of patients had a CRP lab test and $92.2 \%$ had an ESR lab test. When the test value that immediately preceded the GCA diagnosis was of Diseases, Ninth Revision, Clinical Modification, ICD10-CM International Classification of Diseases, Tenth Revision, Clinical Modification

analyzed, mean CRP was $14.9 \mathrm{mg} / \mathrm{dL}$ and mean ESR was $46.7 \mathrm{~mm} / \mathrm{h}$.

During the 12-month post-index period, nearly three-quarters $(74.4 \%)$ of patients had a CRP lab test with the mean CRP value of $5.1 \mathrm{mg} / \mathrm{dL}(\mathrm{SD}=13.6)$ and $86.2 \%$ of patients had an ESR lab test with the mean ESR value of $26.5 \mathrm{~mm} / \mathrm{h}(\mathrm{SD}=20.7)$.

\section{Oral Glucocorticoid Dosing}

The mean daily dose of oral GC of the entire cohort was $28.9 \mathrm{mg}(\mathrm{SD}=23.0)$ during the first 6-month post-index period. Patient stratification by quartiles of oral GC daily dose showed 184 patients each in $\mathrm{Q} 1$ ( $\geq 1.00$ to $\leq 13.75 \mathrm{mg})$ 
Table 1 Baseline patient characteristics

All patients $(N=785)$

Age (years), mean (SD)

$75.8(8.6)$

Age group (years), $N$ (\%)

$50-59$
$60-69$
$70-79$
$80-89$

$42(5.4 \%)$

$139(17.7 \%)$

$266(33.9 \%)$

Sex, $N(\%)$

Male

$239(30.4 \%)$

Female

$546(69.6 \%)$

Geographic region, $N(\%)$

Northeast

$75(9.6 \%)$

North Central

$459(58.5 \%)$

South

$143(18.2 \%)$

West

$107(13.6 \%)$

Unknown

$1(0.1 \%)$

DCCI, mean $(\mathrm{SD})$

$1.6(2.0)$

DCCI comorbidities, $N(\%)$

Diabetes (mild to moderate)

$194(24.7 \%)$

Cerebrovascular disease

$150(19.1 \%)$

Rheumatologic disease other than systemic lupus erythematosus and rheumatoid arthritis ${ }^{\mathrm{a}}$

$148(18.9 \%)$

Polymyalgia rheumatica

$135(17.2 \%)$

Renal disease

$89(11.3 \%)$

Chronic pulmonary disease

$87(11.1 \%)$

Congestive heart failure

$72(9.2 \%)$

Malignancy

$65(8.3 \%)$

Peripheral vascular disease

$58(7.4 \%)$

Diabetes with chronic complications

$54(6.9 \%)$

Conditions indicative of GCA severity, $N(\%)$

Vision impairment including blindness

$89(11.3 \%)$

Aortic aneurysm

$19(2.4 \%)$

Laboratory tests prior to GCA diagnosis

CRP, $N(\%)$

$571(72.7 \%)$

Mean test result, $\mathrm{mg} / \mathrm{dL}(\mathrm{SD})$

$14.9(36.9)$ 
Table 1 continued

All patients $(N=785)$

ESR, $N(\%)$

$724(92.2 \%)$

Mean test result, $\mathrm{mm} / \mathrm{h}(\mathrm{SD})$

$46.7(32.8)$

$D C C I$ Deyo-Charlson comorbidity index, GCA giant cell arteritis, $S D$ standard deviation

${ }^{a}$ Rheumatologic disease includes polymyositis, Felty's syndrome, rheumatoid lung, and polymyalgia rheumatica

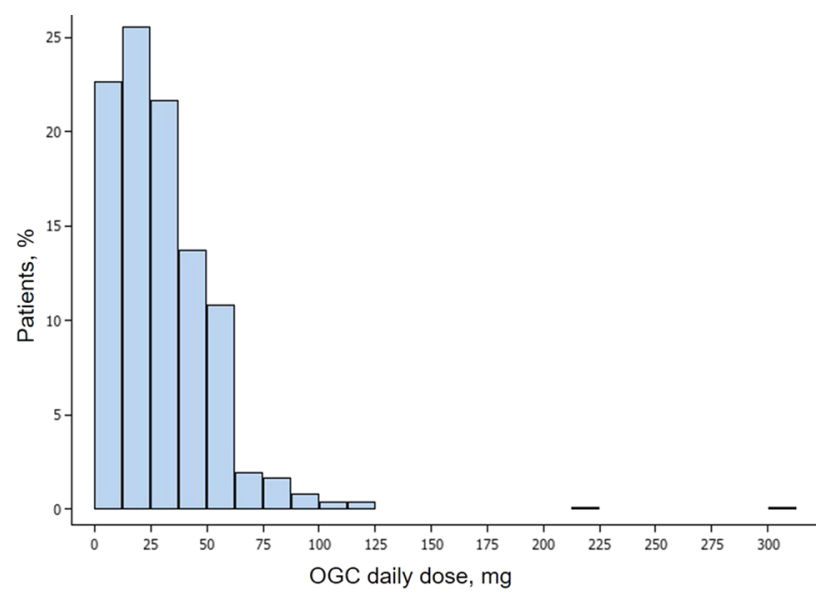

Fig. 2 Distribution of daily oral glucocorticoid dose in milligrams (mg)

and Q2 (>13.75 to $\leq 25.00 \mathrm{mg})$ cohorts, 198 patients in Q3 cohort ( $>25.00$ to $\leq 40.00 \mathrm{mg}$ ), and 161 patients in Q4 cohort $(>40.00 \mathrm{mg}$ ) (Fig. 2). Approximately half (47.0\%) of the patients had a daily oral GC dose of at least $40 \mathrm{mg}$ on or within 30 days following the index date. Of these patients, $79.4 \%$ had a subsequent decrease of oral GC daily dose to below $40 \mathrm{mg}$ within a mean of 84.9 days $(S D=69.6)$. Within the 12-month follow-up period, $17.4 \%$ of these patients had a subsequent increase in the oral GC dose to at least $40 \mathrm{mg}$ in a mean of 84.0 days $(\mathrm{SD}=83.9)$.

\section{Potential Glucocorticoid-Related Adverse Events}

Figure 3 shows the prevalence of endocrine/ metabolic AEs. Among those without T2D or $\mathrm{HbA} 1 \mathrm{c} \geq 7.5 \%$ in the pre-index period, the proportion of patients with newly diagnosed T2D or HbA1c $\geq 7.5 \%$ during the 12 -month follow-up ranged from $7.5(\mathrm{Q} 1)$ to $24.5 \%(\mathrm{Q} 4)$.
Among patients with T2D and controlled HbA1c in the pre-index period, the proportion of patients with worsening diabetes increased from $32.3 \%$ in the Q1 cohort to $50.0 \%$ in the Q3 cohort, prior to decreasing to $26.7 \%$ in the Q4 cohort. The maximum HbA1c value across HbA1c lab results during the 12-month followup was on average $7.8(\mathrm{SD}=2.5)$ in $\mathrm{Q} 1$ and increased to $8.3(\mathrm{SD}=2.9), 8.2(\mathrm{SD}=2.4)$, and $8.6(\mathrm{SD}=3.1)$ in the $\mathrm{Q} 2, \mathrm{Q} 3$, and $\mathrm{Q} 4$ cohorts, respectively. When patients who did not have a lab test with blood glucose level $\geq 200 \mathrm{mg} / \mathrm{dL}$ in the pre-index period were examined, the proportion of patients with glucose $\geq 200 \mathrm{mg} /$ $\mathrm{dL}$ in the follow-up period increased across daily dose quartiles from $7.5 \%$ in Q1 to $15.0 \%$ in Q4. Similarly, among patients with no diagnoses of impaired fasting glucose, impaired glucose tolerance, or abnormal glucose and no lab tests with blood glucose level $\geq 200 \mathrm{mg} / \mathrm{dL}$ in the pre-index period, we noted an increase in glucose intolerance based on diagnoses or lab tests during the post-index period from $16.0 \%$ in Q1 to $26.7 \%$ in Q4. An increase in BMI $\geq 5$ units 


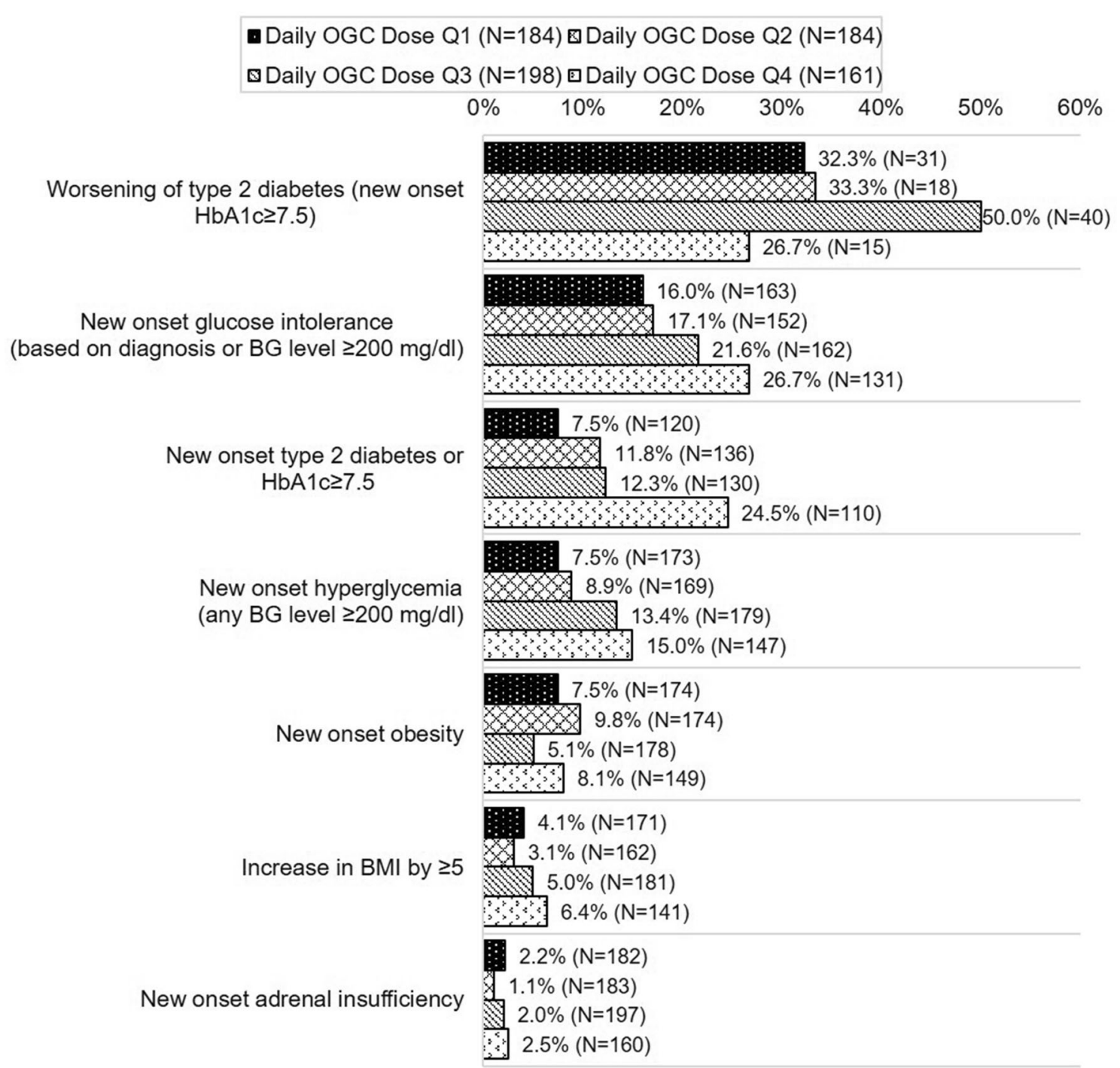

Fig. 3 Prevalence of metabolic and endocrine-related adverse events. ${ }^{*}$ Mean daily dose: Q1, $\geq 1.00$ to $\leq 13.75 \mathrm{mg} ; \mathrm{Q} 2,>13.75$ to $\leq 25.00 \mathrm{mg} ; \mathrm{Q} 3, \geq 25.00$ to $\leq 40.00 \mathrm{mg} ; \mathrm{Q} 4, \geq 40.00 \mathrm{mg}$. For all the new onset events, the total number of patients without the event in

ranged from 3.1\% in the Q2 cohort to $6.4 \%$ in the Q4 cohort.

Figure 4 shows the prevalence of other AEs. The proportions of patients with a new bonerelated AE (osteoporosis or fracture diagnosis) in the post-index period were as follows: Q1: 22.8\%; Q2: 17.4\%; Q3: 23.5\%; Q4: $19.4 \%$. Among those without an osteoporosis medication during the pre-index period, the proportion of patients with osteoporosis medications the pre-index period (i.e., the denominator for the proportions) is presented as " $\mathrm{N}$ " in the figure. HbAlc hemoglobin Alc, BG blood glucose, OGC oral glucocorticoid, OGTT oral glucose tolerance test, Q quartile

during follow-up increased from $29.4 \%$ in the Q1 cohort to $41.8 \%$ in the Q3 and $37.1 \%$ in the Q4 cohorts.

During the 12-month post-index period, the prevalence of serious infections ranged from $16.8 \%$ to $24.8 \%$ from the Q1 to the Q4 cohort. Similarly, the prevalence of serious infections requiring intravenous antibiotics also increased from $15.8 \%$ in the Q1 cohort to $21.7 \%$ in the Q4 cohort. The proportion of patients diagnosed 


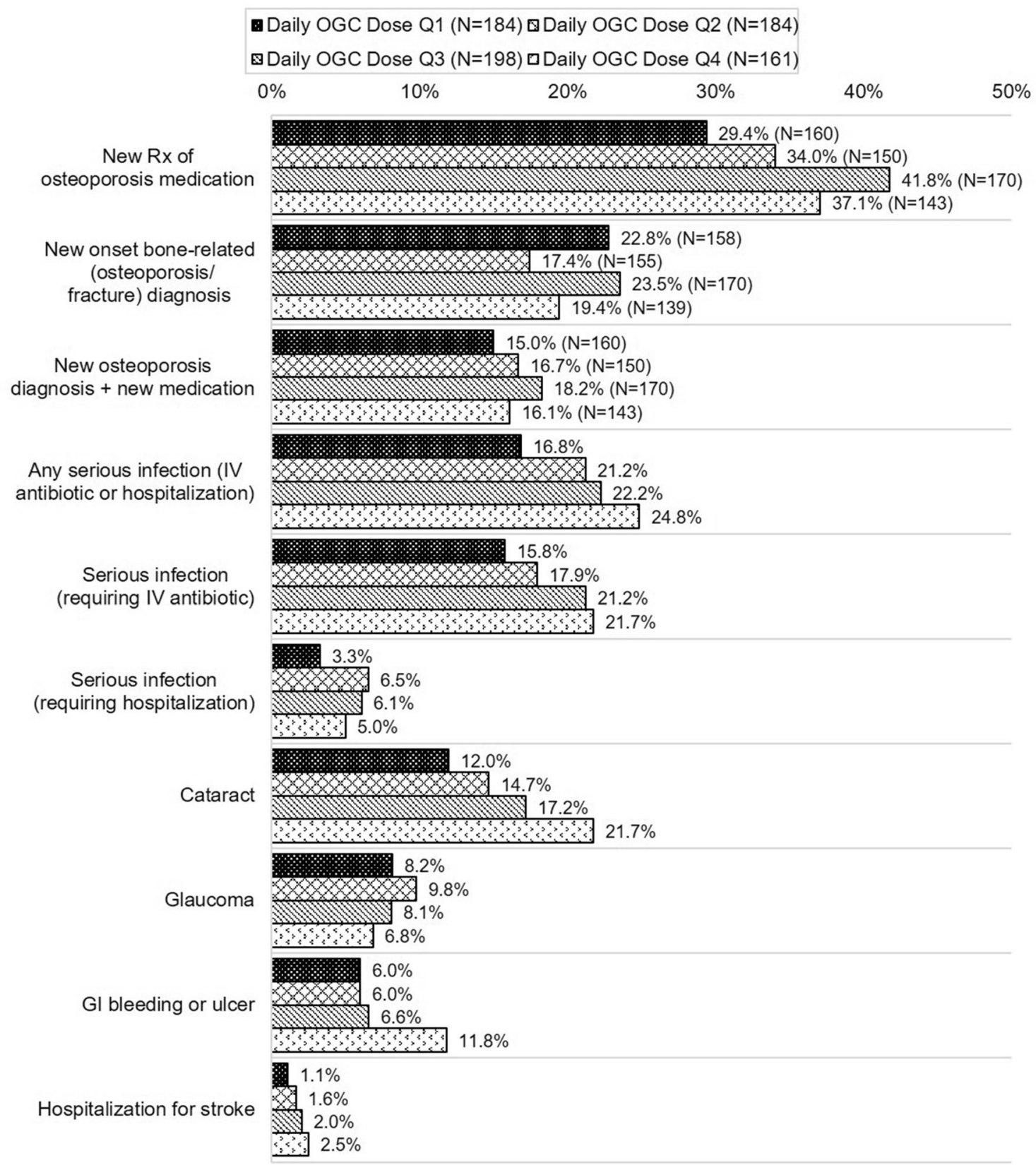

Fig. 4 Prevalence of other adverse events. * Mean daily dose: Q1, $\geq 1.00$ to $\leq 13.75 \mathrm{mg} ; \quad$ Q2, $>13.75$ to $\leq 25.00 \mathrm{mg} ; \quad \mathrm{Q} 3, \geq 25.00$ to $\leq 40.00 \mathrm{mg} ; \quad$ Q 4, $\geq 40.00 \mathrm{mg}$. For all the new onset events, the total number of patients without the event in the pre-index period (i.e., the denominators for the proportions) is presented as " $\mathrm{N}$ " in the figure. Where not reported,

with cataracts increased by nearly $10 \%$ in the Q4 cohort (21.7\%) compared to the Q1 cohort $(12.0 \%)$. A larger proportion of patients in the reference the overall quartile Ns presented in the legend. During the follow-up period, only eight patients had a diagnosis of Cushing's disease and only one patient had a hospitalization for myocardial infarction during the follow-up; therefore, these outcomes are not reported in the figure. GI gastrointestinal, IV intravenous, OGC oral glucocorticoid, Q quartile

Q4 cohort developed GI bleeding or ulcer compared to patients in the Q1 cohort. An inconsistent trend was noted across quartiles in 
patients diagnosed with glaucoma. Though rare, the proportions of patients with a hospitalization for stroke increased from the Q1 to the Q4 cohort.

\section{DISCUSSION}

Prolonged exposure to GC significantly increases the risk of an array of AEs in patients with GCA. In this study, we estimated the risk of steroid toxicity in patients with GCA by analyzing the outcomes of more than 700 patients treated with oral GC. Our results indicate that diabetes, worsening glucose metabolism, cataracts, and serious infections occur frequently in this patient population. Moreover, GC-related AEs occurred soon after GCA diagnosis (within 1 year) and generally in a dose-dependent manner. While the prevalence of GC toxicity in GCA differs by study depending on the methodology used, including the definition of AEs and the duration of follow-up, the patterns of oral GC use and AEs in the patients with GCA in our study are largely consistent with prior data presented in the literature [7-14]. However, by incorporating laboratory results and BMI information for better characterization of the outcomes of interests, our analysis adds to the existing body of work on the subject of GC toxicity.

We found that the average daily oral GC dose was associated with several negative outcomes related to glucose metabolism including new onset T2D (seen in up to $24.5 \%$ of Q4 patients), hyperglycemia (seen in up to $15 \%$ of Q4 patients), and glucose intolerance (seen in up to $26.7 \%$ of Q4 patients). Worsening preexistent diabetes occurred in up to $50 \%$ of patients, but did not clearly parallel the average daily GC dose. Other analyses have reported similar findings [7, 10, 12, 13]. Researchers using both US and UK data found that both glucose intolerance and diabetes were associated with higher GC exposure [12]. For every 1-g increase in cumulative GC dose over 1 year, there was a $7-14 \%$ increase in the odds of glucose intolerance and a $12 \%$ increase in the odds of diabetes [12]. In another US claims-based analysis, the risk of new onset diabetes increased by $5 \%$ with each additional gram of GC intake [7]. In contrast to prior studies, our definition of AEs related to glucose metabolism included key laboratory values (e.g., HbA1c and serum glucose) that most likely increased the accuracy with which these complications were captured.

How GCs impact BMI in patients with GCA has been underexplored. We were able to examine this outcome and found that the proportion of patients with GCA experiencing an increase in BMI of 5 units tended to be higher among those receiving higher daily GC doses (>6\% in Q4 patients). Assessment of BMI has been limited in large observational studies because weight and height information is generally not available in claims, a commonly used data source. Our findings related to BMI changes with the use of GC are important given the implication of BMI in cardiovascular risk, which is known to be increased in elderly patients in general and in patients with GCA in particular [15].

In agreement with work done by others, we observed increased incidence of infection in patients with GCA receiving higher GC doses $[7,10,12]$. In fact, $25 \%$ of patients with GCA in Q4 who were receiving a mean daily GC dose of $40 \mathrm{mg}$ or more developed a serious infection that required hospitalization and/or intravenous antibiotic therapy. In addition, other GC-related AEs including cataracts and GI bleeding or ulcer showed a pattern of incidence that mirrored the average daily GC dosing.

Several studies have reported that patients with GCA exposed to GC are at greater risk for metabolic bone disease including osteoporosis and fragility fracture [7, 10, 11, 14]. In our cohort, approximately $20 \%$ of the patients received the diagnosis of osteoporosis or fracture. Nevertheless, we did not demonstrate a clear GC dose-related trend in the incidence of this complication. One speculation for the absence of a dose-dependent effect could be that 1 year of follow-up might not be a sufficiently long period of time to fully capture the impact of different GC doses on bone metabolism. Studies with longer longitudinal monitoring, however, have reported associations 
between cumulative GC exposure and risk of osteoporosis and fracture [7, 12, 14].

Some limitations of this study merit consideration. This study was limited to individuals seeking care with providers covered by the Explorys healthcare system. Any contact these patients may have had with healthcare providers outside of Explorys EHR could not be captured. Consequently, missing data could have introduced bias to our analysis. In some cases, the number of patients eligible to experience a certain outcome in each quartile was small, precluding us from drawing strong conclusions. The potential for misclassification of GCA and AEs exists as the data for this study were derived from EHR, which are subject to data coding limitations and data entry error. However, any misclassification is expected to be balanced across GC dose quartiles given the large sample size. Patients may have been prescribed other medications to treat their disease, such as methotrexate, or to treat comorbid conditions that may confound the associations between GC dose and AEs. Levels of GCA disease activity and inflammation, which may also be associated with some of the AEs evaluated in this analysis, were not available in the EHR system. Finally, patients were assumed to fill and take their medications as prescribed, but the actual GC use by patients could not be confirmed. We were unable to calculate cumulative GC dose because the length of prescription was not available for all GC prescriptions and length of prescription is required for cumulative dose calculation. The database does not contain dose information for intravenous GC administrations.

\section{CONCLUSION}

Current treatment recommendations call for the use of high doses of GC to treat GCA $[16,17]$. However, consistent with other analyses, we found that an increase in the oral GC dose increases the risk of several glucocorticoidrelated AEs $[5,6,10,12]$. Potential GC-related AEs were more prevalent in patients with GCA who were prescribed higher doses of oral GC and the risk of AEs increased systematically with the increase in daily GC dose. Understanding the patterns of GC-related AEs may drive the exploration of additional treatment options. Effective GC-sparing therapies should be encouraged to reduce the exposure and potential risk of GC and improve clinical outcomes in patients with GCA. Additionally, these findings highlight the need for newer GC-sparing treatment options that could reduce the clinical burden imposed by GC-related AEs among patients with GCA.

\section{ACKNOWLEDGEMENTS}

Funding. This study and the journal's Rapid Service Fee were funded by Genentech, Inc.

Medical Writing and Other Assistance. Input on study endpoints provided by Christine Birchwood and Tien Nguyen of Genentech, Inc. Medical writing services, provided by Matthew Brouillette and Santosh Tiwari of IBM Watson Health, were funded by Genentech, Inc.

Authorship. All named authors meet the International Committee of Medical Journal Editors (ICMJE) criteria for authorship for this article, take responsibility for the integrity of the work as a whole, and have given their approval for this version to be published.

Disclosures. Jennie H. Best is an employee and stockholder of Genentech, Inc. Margaret Michalska is an employee and stockholder in Genentech, Inc. Amanda M. Kong is an employee of IBM Watson Health which received funding to conduct this analysis. Oth Tran is an employee of IBM Watson Health which received funding to conduct this analysis. Sebastian Unizony is an employee of Massachusetts General Hospital and Harvard Medical School.

Compliance with Ethics Guidelines. All database records are anonymized and fully compliant with US patient confidentiality requirements, including the Health Insurance Portability and Accountability Act (HIPAA) of 1996. Because this study did not involve the 
collection, use, or transmittal of individually identifiable data, institutional review board (IRB) approval was not required.

Data Availability. The datasets generated and/or analyzed during the current study are proprietary and are therefore not publicly available. Information on the Explorys data and analytics solutions can be found at https:// www.ibm.com/watson-health/explorys.

Open Access. This article is distributed under the terms of the Creative Commons Attribution-NonCommercial 4.0 International License (http://creativecommons.org/licenses/ by-nc/4.0/), which permits any noncommercial use, distribution, and reproduction in any medium, provided you give appropriate credit to the original author(s) and the source, provide a link to the Creative Commons license, and indicate if changes were made.

\section{REFERENCES}

1. Winkler A, True D. Giant cell arteritis: 2018 review. Mo Med. 2018;115(5):468-70.

2. De Smit E, Palmer AJ, Hewitt AW. Projected worldwide disease burden from giant cell arteritis by 2050. J Rheumatol. 2015;42(1):119-25.

3. The Johns Hopkins Vasculitis Center. Giant cell arteritis. The Johns Hopkins Vasculitis Center; 2019. http://www.hopkinsvasculitis.org/typesvasculitis/giant-cell-arteritis/. Accessed 22 Aug 2019.

4. Crowson CS, Matteson EL. Contemporary prevalence estimates for giant cell arteritis and polymyalgia rheumatica, 2015. Semin Arthritis Rheum. 2017;47(2):253-6.

5. Buttgereit F, Matteson EL, Dejaco C, Dasgupta B. Prevention of glucocorticoid morbidity in giant cell arteritis. Rheumatol. 2018;57(suppl_2):ii11-21.

6. Nesher G, Sonnenblick M, Friedlander Y. Analysis of steroid related complications and mortality in temporal arteritis: a 15-year survey of 43 patients. J Rheumatol. 1994;21(7):1283-6.

7. Broder MS, Sarsour K, Chang E, et al. Corticosteroid-related adverse events in patients with giant cell arteritis: a claims-based analysis. Semin Arthritis Rheum. 2016;46(2):246-52.

8. Proven A, Gabriel SE, Orces C, O'Fallon WM, Hunder GG. Glucocorticoid therapy in giant cell arteritis: duration and adverse outcomes. Arthritis Rheum. 2003;49(5):703-8.

9. Fraser JA, Weyand CM, Newman NJ, Biousse V. The treatment of giant cell arteritis. Rev Neurol Dis. 2008;5(3):140-52.

10. Wilson JC, Sarsour K, Collinson N, et al. Serious adverse effects associated with glucocorticoid therapy in patients with giant cell arteritis (GCA): a nested case-control analysis. Semin Arthritis Rheum. 2017;46(6):819-27.

11. Curtis JR, Westfall AO, Allison J, et al. Populationbased assessment of adverse events associated with long-term glucocorticoid use. Arthritis Rheum. 2006;55(3):420-6.

12. Gale S, Wilson JC, Chia J, et al. Risk associated with cumulative oral glucocorticoid use in patients with giant cell arteritis in real-world databases from the USA and UK. Rheumatol Ther. 2018;5(2):327-40.

13. Faurschou M, Ahlstrom MG, Lindhardsen J, Obel N, Baslund B. Risk of diabetes mellitus among patients diagnosed with giant cell arteritis or granulomatosis with polyangiitis: comparison with the general population. J Rheumatol. 2017;44(1):78-83.

14. Paskins Z, Whittle R, Sultan AA, et al. Risk of fracture among patients with polymyalgia rheumatica and giant cell arteritis: a population-based study. BMC Med. 2018;16(1):4.

15. Tomasson G, Peloquin C, Mohammad A, et al. Risk for cardiovascular disease early and late after a diagnosis of giant-cell arteritis: a cohort study. Ann Intern Med. 2014;160(2):73-80.

16. Dasgupta B, Borg FA, Hassan N, et al. BSR and BHPR guidelines for the management of giant cell arteritis. Rheumatol. 2010;49(8):1594-7.

17. Warrington KJ, Matteson EL. Management guidelines and outcome measures in giant cell arteritis (GCA). Clin Exp Rheumatol. 2007;25(6 Suppl 47):137-41. 